\title{
Traffic Signal Coordination for Tramlines with Passive Priority Strategy
}

\author{
Yun Bai $\mathbb{D}^{1},{ }^{1}$ Jiajie Li $(\mathbb{D}),{ }^{1}$ Tang Li $\left(\mathbb{D},{ }^{2}\right.$ Lingling Yang, ${ }^{3}$ and Chenxi Lyu ${ }^{4}$ \\ ${ }^{1}$ MOE Key Laboratory for Urban Transportation Complex Systems Theory and Technology, Beijing Jiaotong University, No. 3, \\ Shangyuancun, Haidian District, Beijing 100044, China \\ ${ }^{2}$ Department of Civil and Environmental Engineering, Imperial College London, London SW7 2AZ, UK \\ ${ }^{3}$ Stantec Consulting Services, Inc., Denver, CO 80222, USA \\ ${ }^{4}$ School of Economics and Management, Ningbo University of Technology, Zhejiang, Ningbo 315211, China
}

Correspondence should be addressed to Jiajie Li; 15120834@bjtu.edu.cn

Received 29 July 2018; Accepted 6 November 2018; Published 22 November 2018

Academic Editor: Mahmoud Mesbah

Copyright (c) 2018 Yun Bai et al. This is an open access article distributed under the Creative Commons Attribution License, which permits unrestricted use, distribution, and reproduction in any medium, provided the original work is properly cited.

Prioritizing traffic signals for trams crossing intersections without stops can increase the service punctuality and travel speed of trams, but it may also increase the delays of other vehicles at intersections. This paper presents a model on coordinated control of traffic signals among successive intersections along the tramline, taking into account driving characteristics of trams and vehicles. The objective is maximizing the valid bandwidth of vehicle green wave to reduce vehicle delays, while the trams cross intersections without stops. Linear Interactive and General Optimizer (LINGO) is applied to solve the proposed model and VISSIM simulation software is adopted to assess the solutions attained by the proposed model and the previous TRAMBAND model. Case studies show that the solutions given by the proposed model facilitate trams to go through all intersections along the tramline without stops. In comparison with the TRAMBAND model, the proposed model reduces tram delay by $13.14 \mathrm{~s} / \mathrm{pcu}$ and increases the throughput of vehicles at intersections by $4.45 \%$ and reduces vehicle delays by $2.22 \%$. Extensive simulations have verified that the performance of the proposed model is stable under different tram headways, dwell time, and traffic volumes. It is also found that the tram headway must be multiple of traffic signal cycle time to completely realize green wave control of all trams at all intersections along the tramline.

\section{Introduction}

As one of urban rail transit modes, modern tram is becoming an important part of public transportation especially in medium-sized cities, due to its advantages of low investment and environment protection. At present, there are hundreds of cities across the world that operate tram systems. In most cities, trams run in the inner lane of the road and they have the exclusive rights of way except for intersections, where they share the rights of way with vehicles which refer to all automobiles outside of trams. Allocating exclusive lanes can reduce travel time by lessening possible disturbances $[1,2]$. However, trams have to stop in front of intersections if they hit red signals. In this situation, tram travel time and energy consumption increase remarkably as stops need braking and reacceleration. It is necessary to give trams a prior right of way at intersections to avoid unplanned stops $[3,4]$.

Generally, there are three major strategies to provide trams with prior signals at intersections. The first one is active priority giving green traffic signals to trams whenever trams approach intersections. The measures of active priority include green phase extension, red phase reduction, green phase insertion, and giving an exclusive phase for trams [58]. However, active priority generally results in the heaviest delays on the vehicle and pedestrian traffic among all the three strategies. And, detectors must be installed ahead of intersections to activate the tram priority, which involves significant investment. The second strategy is passive priority (also called green wave control), which creates a green wave band for tram crossings at successive intersections by adjusting the parameters of traffic signals such as phase 
sequences and shifts [9-11]. With passive priority, detectors are not required for implementation and the impact on vehicle and pedestrian traffic could be minimized [12]. The practical performance of passive priority is governed by the reliability of tram run-time between intersections. Trams usually adopt semiexclusive right of way and are equipped with the Automatic Operation Control Systems [13], which enables accurate control of tram run-time on road segments and in turn facilitates the passive priority signal control on tramlines. The last one is adaptive priority to optimize signal timing plan according to the real-time traffic volumes [1417], which also involves substantial investment on detectors as well as heavier delays on vehicles in comparison with passive priority. The adaptive priority has been explored on buslines where it is impractical to control bus run-time between intersections accurately $[18,19]$.

Among these strategies, passive priority is commonly adopted to realize the green wave control of vehicles and public transits, due to relatively low cost and less impact on vehicle and pedestrian traffic. MAXBAND, MULTIBAND, and AM-BAND are three classical models of signal time planning with passive priority. MAXBAND formulates a mixed integer linear programming model to find the optimal signal timing parameters, such as cycle length, offset, phase time, and travel speed, to maximize the bandwidth of green wave [20-22]. The green wave bands generated by MAXBAND are bilateral and symmetrical and they have the same width in different road sections. Relaxing the constraint of equal bandwidth at different sections, MULTIBAND is able to expand the width of green wave band of MAXBAND [2325]. AM-BAND further releases the symmetrical constraint on green wave band, to improve the utilization of green time and decrease vehicle delays at intersections [26]. The earlier models did not take into account the dwell time at stations, which are inapplicable to buses and trams.

In recent years, several improved models were proposed for passive priority signal control of public transits, considering the dwell time at stations [27]. Dai et al. proposed a new MAXBAND-based model to find the optimal signal timing plan in consecutive intersections to facilitate the green wave control of both buses and other vehicles [28]. Jeong and Kim developed a TRAMBAND model to increase the green bandwidth of vehicles while keeping the bandwidth of trams as fixed [29]. However, the TRAMBAND model is based on MAXBAND model, which is not as good as AM-BAND model in maximizing green wave bandwidth. In other words, there is still space to enlarge the vehicle green bandwidth generated by the TRAMBAND model. Based on AM-BAND model, Zhou et al. established a BAM-TRAMBAND model to maximize the valid bandwidth of green wave for trams, considering a part of the green wave generated by AMBAND model could not be used to allow trams crossing intersections without stops [30]. The objective function of BAM-TRAMBAND model does not consider vehicle delays at intersections. In real practice, tram headway is usually larger than the cycle length of intersection traffic signals, which means there is only one tram crossing an intersection within one cycle of traffic signal. And, a tram only needs a few seconds to go through an intersection. As a result, there is no need to maximize the green bandwidth for trams. Instead, it is of more significance to maximize the vehicle green bandwidth, while the tram bandwidth is large enough to ensure trams pass through intersections without stops.

There are three flaws of the previous studies in passive priority on tramlines. First, the previous studies assumed that trams always travel at a constant speed recommended by the green wave band. In real world, tram must decelerate to approach a station and then accelerate to attain the full operation speed, which indicates time loss in comparison with the fact that the tram travels at a constant speed. The time loss for each station stop is normally as large as 20 seconds. Without considering the time loss in optimizing the signal timing plans, trams might arrive at intersections beyond the planned green wave band and hit red signals. Second, the vehicle speed recommended by the green wave band from previous studies may vary in different sections. However, it is impractical for drivers controlling vehicles to strictly follow the recommended speed as it changes in different sections. To ensure the effectiveness of the passive priority, the driving characteristics of vehicles and trams should be considered in the traffic signal timing. Finally, the valid bandwidth of green wave in previous studies has not been analysed thoroughly in previous studies, which might still result in invalid green time.

In this study, an improved model based on AM-BAND is presented for passive priority signal control in a bidirectional tramline, to maximize the green wave bandwidth of vehicles while maintaining the green wave control of trams. The possible reasons of invalid green bandwidth are analysed systematically and controlled out of the optimization. The proposed model takes into account the time loss caused by tram deceleration and acceleration at stations and keeps the recommended vehicle speed at different sections constant. In addition, the practical operation constraints on service quality, traction characteristics, and turnaround requirement of trams are fully considered in the proposed model.

The remainder of this paper is organized as follows. Section 2 describes the research problem on signal control of passive priority in a tramline. Section 3 proposes the models on green wave control of vehicles and modern trams, based on the analysis of valid green bandwidth. Section 4 gives case studies to investigate the performance of the proposed models. Finally, the conclusions are summarized in Section 5.

\section{Problem Statement}

The problem discussed in this study is coordinating traffic signals to create a green wave band that allows continuous progressions of trams and vehicles along the corridor as much as possible. Any tram and vehicle travelling within the green wave band at the recommended speed will see a progressive cascade of green lights and will not have to stop at intersections. This allows higher traffic loads and reduces the number of stops, delays, and energy consumption. There are a series of models proposed to achieve the green wave for vehicles by setting appropriate signal cycle time, phase sequence, offset, and vehicle travel speed. The signal coordination becomes complicated when both trams and vehicles 


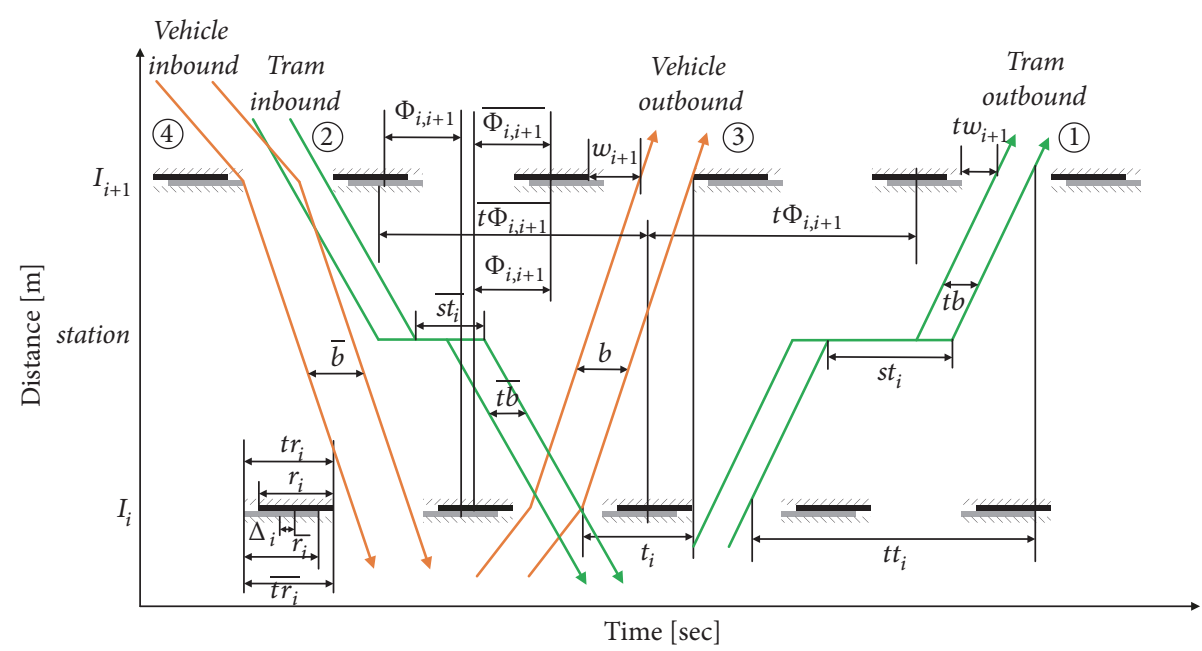

FIGURE 1: Time-space diagram of TRAMBAND model.

are involved because of the difference on vehicle and tram run-times caused by different operation speed and station stop of tram.

TRAMBAND is a signal control optimization model of passive priority, which could provide a balanced signal plan for both trams and vehicles [29]. A typical time-space diagram of TRAMBAND is shown in Figure 1, where the horizontal rectangles represent red signal periods; (1) and (2), respectively, reflect outbound and inbound tram green waves; (3) and (4) are green waves for vehicles in outbound and inbound directions, respectively. TRAMBAND first sets fixed bandwidths for trams and then seeks the maximum bandwidths to provide vehicles with the best progressions along the corridor.

TRAMBAND model has three significant weaknesses. First, the model is built on the MAXBAND model with equal bandwidth at different sections, which limits the utilizing efficiency of green time. For instance, as shown in Figure 1, the left side of green wave (3) still has unused green time. Second, tram acceleration and deceleration time loss is not included in the model. The accumulated time loss would shift the actual tram trajectory, which means the tram is very likely to meet a red signal in following intersections. For example, the accumulated time loss will shift actual operation trajectory of (1) to the right side. If the accumulated time loss is big enough, trams will meet the red signal at $I_{i+1}$ intersection even when operated at the recommended speed. Third, to maximize vehicles' bandwidth, the TRAMBAND model released the constraint that the recommended speed for vehicles in different sections should be constant. Different recommended speeds at different sections increase the complexity of vehicle driving because drivers have to adjust the operation speed frequently. For example, the recommended speeds of green wave (3) before and after intersection $I_{i}$ are different. If the driver does not change vehicle speed, the vehicle will meet the red signal at intersection $I_{i+1}$.

To overcome the weaknesses of TRAMBAND model, this paper proposes an improved model for passive priority signal control in tramlines based on AM-BAND model which can provide better utilization of green time in comparison with MAXBAND and MULTBAND models. The proposed model aims to find the optimal cycle time, phase sequence, offset of traffic signals, and recommended travel speed, without changing the green split, i.e., the ratio of effective green time to the cycle length. The objective is to maximize the valid bandwidth of vehicles' green wave, while trams' green bandwidth is preset ensuring the green wave control of trams. The proposed model takes time loss for tram deceleration and acceleration before and after stops into account. Meanwhile, the recommended speed for vehicles is fixed, which allows drivers to easily follow the green wave band. However, tram drivers could remember the operation speed recommendation in different sections because they drive along the line every day. As a result, this study does not fix the recommended speed of trams, which provides more optimisation space for vehicles' bandwidth.

\section{Modelling}

This section formulates a traffic signal coordinate model to maximize vehicles' green bandwidth constrained by preset trams' green band, which allows trams cross intersections without a stop. The model consists of vehicles part and trams part as follows.

\subsection{Improved AM-BAND Model for Vehicles. AM-BAND is} a signal coordinate model to find two-way green band for vehicle progressions. The time-space diagram of AM-BAND model is shown in Figure 2.

There are $n$ signal-controlled intersections along the tramline and $I_{i}$ represents the $i$-th intersection. The time parameters and variables are in unit of the cycle time. The AM-BAND model was formed asin the following equations:

\section{Objective:}

$\max B$ 


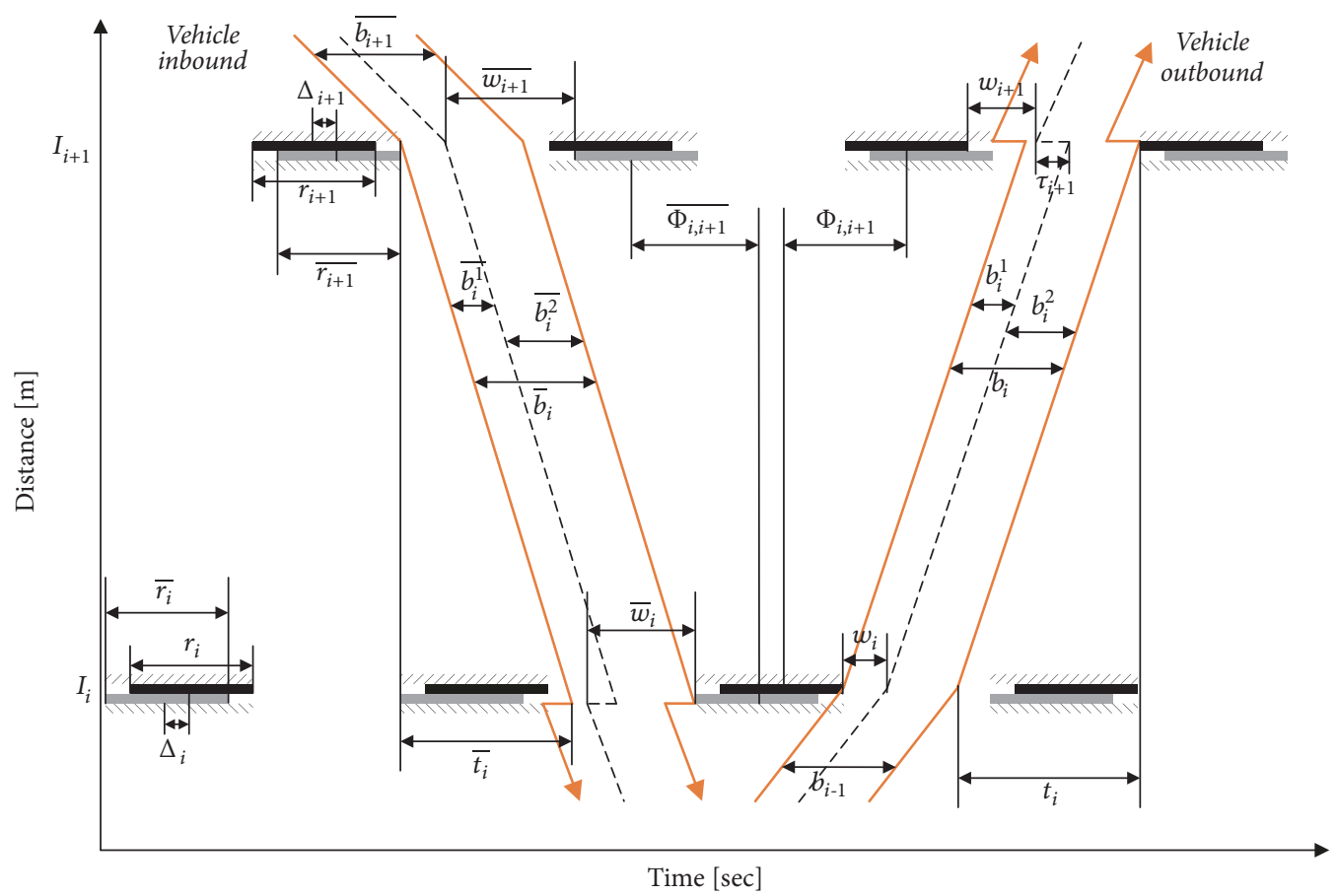

FIGURE 2: Time-space diagram for AM-BAND model.

$=\frac{1}{n-1} \sum_{i=1}^{n-1}\left[a_{i}\left(b_{i}^{1}+b_{i}^{2}\right)+\overline{a_{i}}\left(\overline{b_{i}^{1}}+\overline{b_{i}^{2}}\right)\right]$

(1)

S.t. $\quad\left(1-k_{i}\right) k_{i}\left(b_{i}^{1}+b_{i}^{2}\right) \leq\left(1-k_{i}\right)\left(\overline{b_{i}^{1}}+\overline{b_{i}^{2}}\right)$

$Z_{\min } \leq Z \leq Z_{\max }$

$b_{i}^{1} \leq w_{i} \leq\left(1-r_{i}\right)-b_{i}^{2}$

$b_{i}^{1} \leq w_{i+1}+\tau_{i+1} \leq\left(1-r_{i+1}\right)-b_{i}^{2}$

$\overline{b_{i}^{2}} \leq \overline{w_{i}}-\overline{\tau_{i}} \leq\left(1-\overline{r_{i}}\right)-\overline{b_{i}^{1}}$

$\overline{b_{i}^{2}} \leq \overline{w_{i+1}} \leq\left(1-\overline{r_{i+1}}\right)-\overline{b_{i}^{1}}$ $i=1,2, \cdots, n-1$

$\frac{b_{i}^{2}}{q} \leq b_{i}^{1} \leq q b_{i}^{2}$

$\frac{\overline{b_{i}^{2}}}{q} \leq \overline{b_{i}^{1}} \leq q \overline{b_{i}^{2}}$ $i=1,2, \cdots, n-1$

$$
\begin{aligned}
& \left(t_{i}+\overline{t_{i}}\right)+\left(w_{i}+\overline{w_{i}}\right)-\left(w_{i+1}+\overline{w_{i+1}}\right) \\
& \quad+\delta_{i} L_{i}-\overline{\delta_{i}} \overline{L_{i}}-\delta_{i+1} L_{i+1}+\overline{\delta_{i+1}} \overline{L_{i+1}}+r_{i}
\end{aligned}
$$

$$
\begin{aligned}
-r_{i+1}-\left(\tau_{i+1}+\bar{\tau}_{i}\right) & =m_{i} \\
i & =1,2, \cdots, n-1
\end{aligned}
$$

$$
\begin{gathered}
\frac{d_{i}}{Z \cdot f_{i}} \leq t_{i} \leq \frac{d_{i}}{Z \cdot e_{i}} \\
\frac{\overline{d_{i}}}{Z \cdot \overline{f_{i}}} \leq \overline{t_{i}} \leq \frac{\overline{d_{i}}}{Z \cdot \overline{e_{i}}}
\end{gathered}
$$$$
i=1,2, \cdots, n-1
$$

$$
\begin{aligned}
& \frac{d_{i}}{Z \cdot h_{i}} \leq \frac{d_{i}}{d_{i+1}} t_{i+1}-t_{i} \leq \frac{d_{i}}{Z \cdot g_{i}} \\
& \frac{\overline{d_{i}}}{Z \cdot \overline{h_{i}}} \leq \frac{\overline{d_{i}}}{\overline{d_{i+1}}} \overline{t_{i+1}}-\overline{t_{i}} \leq \frac{\overline{d_{i}}}{Z \cdot \overline{g_{i}}}
\end{aligned}
$$

$$
i=1,2, \cdots, n-2
$$

$b_{i}^{1}, b_{i}^{2}, \overline{b_{i}^{1}}, \overline{b_{i}^{2}}, Z, w_{i}, \overline{w_{i}}, t_{i}, \overline{t_{i}} \geq 0$

$m_{i}$ is integer

$\delta_{i}$ and $\overline{\delta_{i}}$ are $\frac{0}{1}$ dummy variables

Equation (1) is the objective function, which maximizes the weighted average of bandwidth of two directions, denoted by $B . a_{i}\left(\bar{a}_{i}\right)$ is the weight of outbound (inbound) direction 
between intersections $i$ and $i+1$. Normally, $a_{i}\left(\bar{a}_{i}\right)$ depends on the ratio of actual flow to saturation flow of traffic. $b_{i}^{1}\left(\bar{b}_{i}^{1}\right)$ and $b_{i}^{2}\left(\bar{b}_{i}^{2}\right)$ are, respectively, the left and right bandwidths of green waves for outbound (inbound) direction between intersections $i$ and $i+1$.

Equation (2) represents the constraint on ratio between green bandwidths of inbound and outbound directions, which guarantees the minimum bandwidth of the direction with lower traffic demand. $k_{i}=\bar{a}_{i} / a_{i}$ is the ratio of inbound and outbound weights between intersections $i$ and $i+1$.

Equation (3) requires that the cycle time $Z$ is within a reasonable range, where $Z_{\min }$ and $Z_{\max }$ denote the minimum and maximum cycle time, respectively.

Equation (4) claims that green wave must stand in green time area. $w_{i}\left(\bar{w}_{i}\right)$ is the time difference between the outbound (inbound) green wave's centre line and the left (right) side red signal at the section between intersections $i$ and $i+1$. $r_{i}\left(\bar{r}_{i}\right)$ is the red signal period of outbound (inbound) direction at intersection $i . \tau_{i}\left(\bar{\tau}_{i}\right)$ is the clearing time of outbound (inbound) queue at intersection $i$.

Equation (5) guarantees that the bandwidths of both directions are nonzero, and the parameter $q$ can be any positive integer.

Equation (6) establishes the relationship between inbound and outbound directions and integrates two direction green bands into the same time-space diagram. $t_{i}\left(\bar{t}_{i}\right)$ is the traveling time between intersections $i$ and $i+1$ in outbound (inbound) direction. $L_{i}\left(\bar{L}_{i}\right)$ is the left-turn red signal splits at intersection $i$ in outbound (inbound) direction. $\delta_{i}\left(\bar{\delta}_{i}\right)$ equals 1 when the left-turn phase is a lagging phase and equals 0 when the left-turn phase is leading phase; $m_{i}$ is any integer.

Equation (7) requires that vehicle speed falls into the minimum and the maximum speed constrained by driving condition. $d_{i}\left(\bar{d}_{i}\right)$ is the distance between intersections $i$ and $i+1$ in outbound (inbound) direction. $f_{i}\left(\bar{f}_{i}\right)$ and $e_{i}\left(\bar{e}_{i}\right)$ are speed cap and floor between intersections $i$ and $i+1$ in inbound (outbound) direction, respectively.

Equation (8) is the constraint on variation of green wave speed in adjacent sections. $h_{i}\left(\bar{h}_{i}\right)$ and $g_{i}\left(\bar{g}_{i}\right)$ are the cap and floor of speed variation between the adjacent sections before and after intersections $i+1$ in outbound (inbound) direction, respectively. Equations (9) to (11) provide the range of parameters and variables in the model.

Compared to MAXBAND and MULTBAND models, AM-BAND model releases the constraints of equal width and symmetry on green wave bands, which improves the green time utilization. However, the AM-BAND model has two major weaknesses. First, the vehicle normally drives with fixed speed on the main road. AM-BAND model allows variations on recommended vehicle speed in different sections, which increases the difficulty for drivers to follow the green wave. To achieve expectant effect of green wave in practice, this study defines the recommended vehicle speed as fixed in the same direction. Thus, constraints (7) and (8) are substituted by constraint (12).

$$
\begin{aligned}
t_{i} & =\frac{d_{i}}{Z \cdot v} \\
\bar{t}_{i} & =\frac{\overline{d_{i}}}{Z \cdot \bar{v}} \\
& \quad e \leq v \leq f \bar{e} \leq \bar{v} \leq \bar{f} i=1,2, \cdots, n-1
\end{aligned}
$$

In equation $(12) v(\bar{v})$ is the recommended vehicle speed in outbound (inbound) direction; $f(\bar{f})$ and $e(\bar{e})$ are the cap and floor of vehicle speed in outbound and inbound directions, respectively. It should be noted that vehicle speed may fluctuate a little in practice due to mental and physical changes of drivers. The small degree of speed fluctuation has been taken into account in this study from the following two aspects. On one hand, the recommend green wave speed is an average speed, which means it allows the vehicle speed to fluctuate around the fixed value. For example, if the vehicle speed is lower than the recommended one, the driver could speed up in the subsequent running, to make the average speed between intersections close to the recommended value. On the other hand, the width of green wave band allows a small degree of fluctuation on vehicle speed, even if the average speed slightly differs from the recommended speed.

Second, the green wave band generated by AM-BAND model may have different bandwidths in different sections. In this situation, partial green wave band might be ineffective at some intersections. When the bandwidth of green wave is decreasing (Figure 3(a)), the vehicles arriving between $T_{1} \& T_{2}$ or $T_{3} \& T_{4}$ are very likely to meet a red signal at intersection $i+1$ if they follow the recommended speed, which violates the intention of passive priority signal control. As a result, a decreasing bandwidth of vehicle green wave is forbidden in this study.

In another case, an increasing bandwidth allows the vehicles with the speed different from the recommended one that still have a chance to go through the next intersection without a stop. However, the dilated green wave has boundaries to ensure all the green wave band is valid. As in Figure 3(b), $T_{7}$ is the vehicle arrival time at the $i$-th intersection with the maximum speed from $T_{5}$, which is the left border of the valid green wave band at the intersection $i$. Similarly, $T_{10}$ is the vehicle arrival time at the $i$-th intersection with the minimum speed from $T_{6}$, which is the right border of the valid green wave band. In conclusion, the constraint of a valid green band is formulated as follows:

$$
\begin{aligned}
& b_{i+1}^{1} \geq b_{i}^{1} \\
& b_{i+1}^{2} \geq b_{i}^{2} \\
& \overline{b_{i+1}^{1}} \leq \overline{b_{i}^{1}} \\
& \overline{b_{i+1}^{2}} \leq \overline{b_{i}^{2}}
\end{aligned}
$$




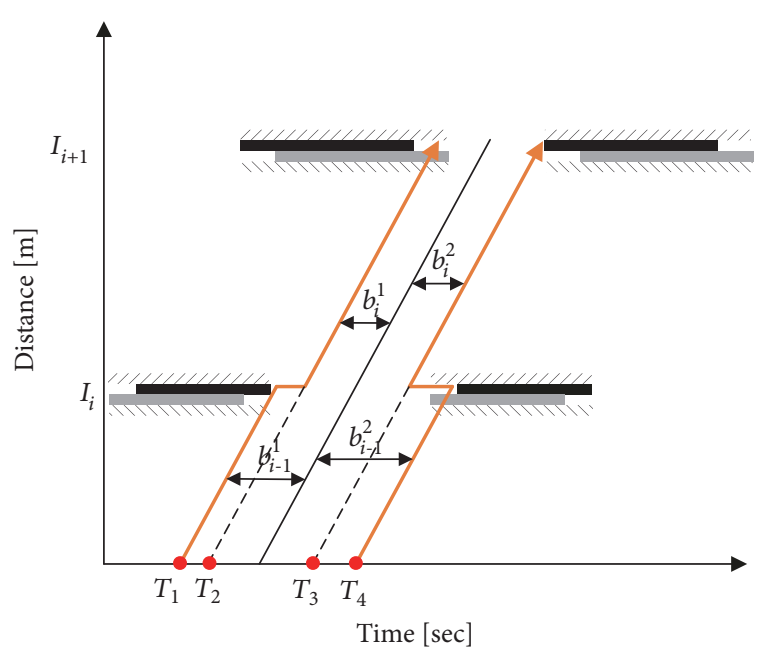

(a) Bandwidth decreases

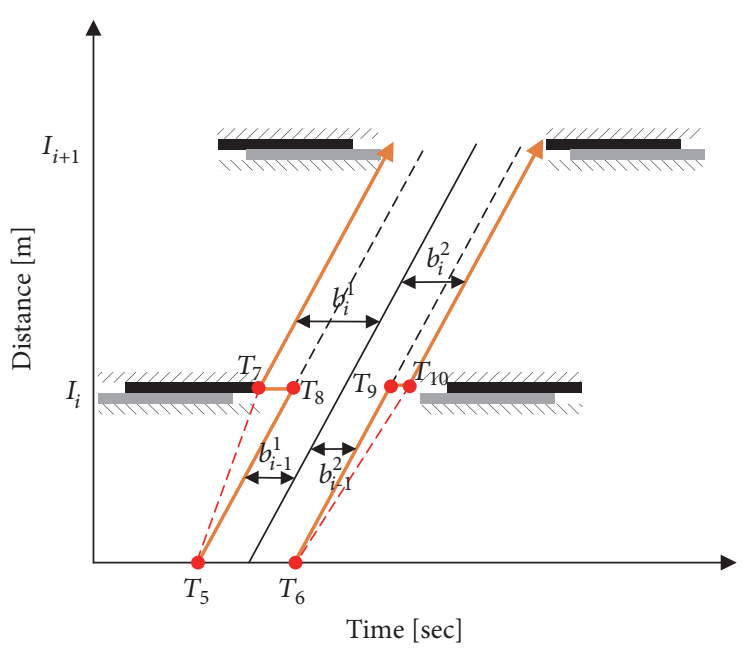

(b) Bandwidth increases

FIgURE 3: Schematic diagram to illustrate the invalid bandwidth of AM-BAND model.

$$
\begin{aligned}
& f \geq \frac{d_{i}}{t_{i}-\left(b_{i+1}^{1}-b_{i}^{1}\right)} \cdot \frac{1}{Z} \\
& \frac{d_{i}}{t_{i}+\left(b_{i+1}^{2}-b_{i}^{2}\right)} \cdot \frac{1}{Z} \geq e \\
& \bar{f} \geq \frac{\overline{d_{i+1}}}{\overline{t_{i+1}}-\left(\overline{b_{i}^{1}}-\overline{b_{i+1}^{1}}\right)} \cdot \frac{1}{Z} \\
& \frac{\overline{d_{i+1}}}{\overline{t_{i+1}}+\left(\overline{b_{i}^{2}}-\overline{b_{i+1}^{2}}\right)} \cdot \frac{1}{Z} \geq \bar{e} \\
& t_{i}-\left(b_{i+1}^{1}-b_{i}^{1}\right)>0 \\
& \overline{t_{i+1}}-\left(\overline{b_{i}^{1}}-\overline{b_{i+1}^{1}}\right)>0 \\
& i=1,2, \cdots, n-2
\end{aligned}
$$

3.2. Signal Coordinate Model for Trams. This section discusses the constraints of signal timing model to ensure the green wave control of trams. The complete signal timing model can be formulated by combining the objective and constraints of vehicles (Section 3.1) and trams (Section 3.2). The time-space diagram for trams is shown in Figure 4. Unless otherwise indicated, the time parameters and variables are in units of the cycle time.

First, trams' green wave should stay in the green period. Trams may have conflicts with left-turn vehicles because trams are usually running in the inner lane of the road. Therefore, the overlap green time between the through phases and the left-turn phases should be removed out of the valid green time for trams. The constraint on the tram green wave is shown as

$$
\begin{array}{rl}
\frac{b_{t}}{2} & \leq w_{t i} \\
\frac{\overline{b_{t}}}{2} & \leq \overline{w_{t i}} \\
w_{t i}+\frac{b_{t}}{2} & \leq 1-\left(r_{i}+\alpha_{i} L_{i}\right) \\
\overline{w_{t i}}+\frac{\overline{b_{t}}}{2} & \leq 1-\left(\overline{r_{i}}+\overline{\alpha_{i} L_{i}}\right) \quad \\
i & i=1,2, \cdots, n-1
\end{array}
$$

where $b_{t}\left(\bar{b}_{t}\right)$ is the green bandwidth of trams in outbound (inbound) direction. $w_{t i}\left(\bar{w}_{t i}\right)$ is the time difference between the centre line of green wave and the left (right) side red signal in outbound (inbound) direction at intersection i. $\alpha_{i}\left(\bar{\alpha}_{i}\right)$ is a zero-one dummy variable. The value is determined by the signal phase sequence at intersection $i$, as shown in

$$
\alpha_{i}=\overline{\alpha_{i}}=\left|\operatorname{sgn}\left(\delta_{i}-\overline{\delta_{i}}\right)\right| \quad i=1,2, \cdots, n-1
$$

where sgn is a Sign function. The return value of Sign function is 0 when $\delta_{i}$ equals $\bar{\delta}_{i}$. The return value of Sign function is 1 when $\delta_{i}$ is larger than $\bar{\delta}_{i}$. The return value of Sign function is -1 when $\delta_{i}$ is smaller than $\bar{\delta}_{i}$.

Second, in order to combine trams and vehicles in both inbound and outbound directions into the same time-space 


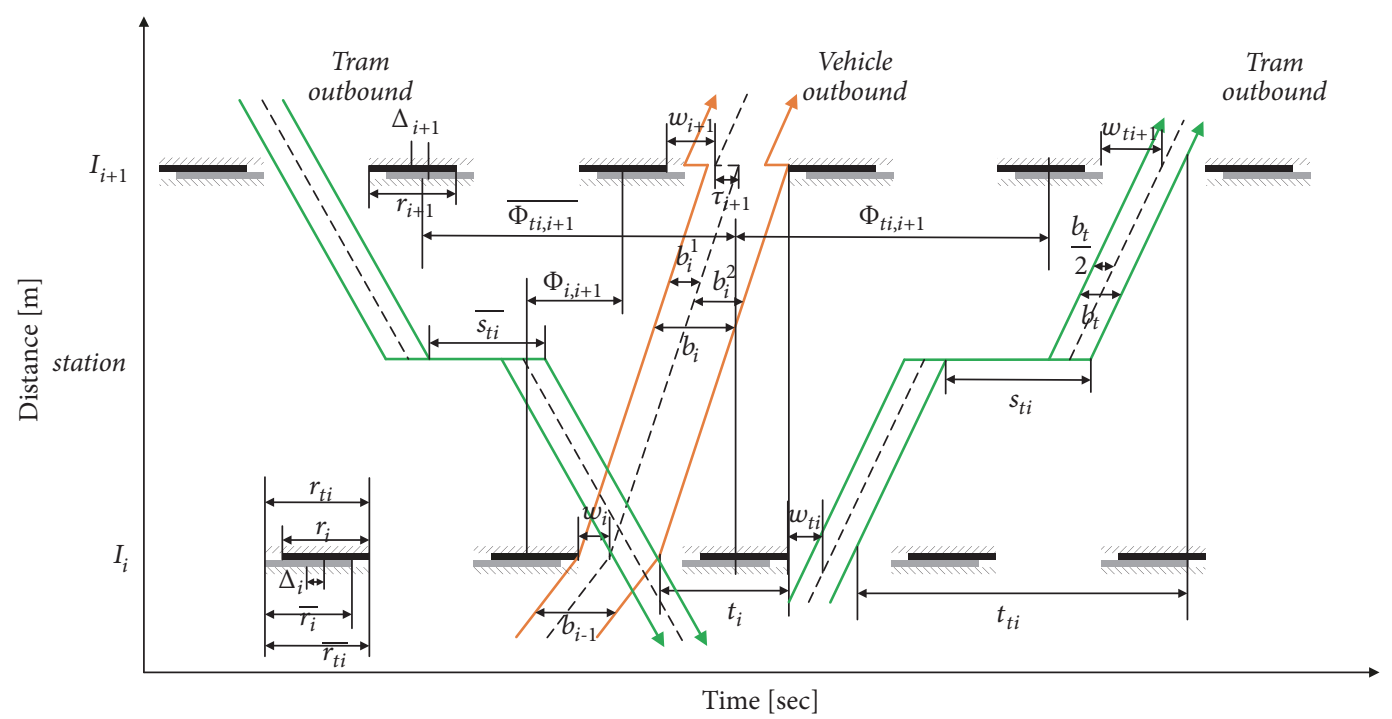

FIgURE 4: Time-space diagram of the tram green wave.

diagram, the green wave for trams and vehicles should satisfy constraints equations (16) and (17).

$$
\begin{aligned}
& \left(t_{t i}+\overline{t_{t i}}\right)+\left(w_{t i}+\overline{w_{t i}}\right)-\left(w_{t i+1}+\overline{w_{t i+1}}\right)+\frac{1}{2} \\
& \cdot\left(r_{i}+\overline{r_{i}}-r_{i+1}-\overline{r_{i+1}}\right)+\frac{1}{2} \\
& \cdot\left[\alpha_{i}\left(L_{i}+\overline{L_{i}}\right)-\alpha_{i+1}\left(L_{i+1}+\overline{L_{i+1}}\right)\right]=m_{t i} \\
& i=1,2, \cdots, n-1 \\
& \left(t_{t i}-t_{i}\right)+\left(w_{t i}-w_{i}+w_{i+1}-w_{t i+1}\right)+\frac{1}{2} \\
& \cdot\left(\alpha_{i} \cdot L_{i}-\alpha_{i+1} \cdot L_{i+1}\right)+\tau_{i+1}-(-1)^{\delta_{i}} \cdot \alpha_{i} \cdot \frac{L_{i}}{2} \\
& +(-1)^{\delta_{i+1}} \cdot \alpha_{i+1} \cdot \frac{L_{i+1}}{2}=q_{t i} \quad i=1,2, \cdots, n-1
\end{aligned}
$$

$t_{t i}\left(\bar{t}_{t i}\right)$ is tram travel time between intersections $i$ and $i+$ 1 in outbound (inbound) direction. $m_{t i}$ and $q_{t i}$ are any integers.

Third, taking safety, service quality and rolling stock characteristics into account, tram speed and its variance should satisfy constraints equations (18) and (19).

$$
\begin{aligned}
& \frac{d_{i}}{Z \cdot f_{t i}}+p_{i} \cdot\left(s_{t i}+C_{t i}\right) \leq t_{t i} \leq \frac{d_{i}}{Z \cdot e_{t i}}+p_{i} \cdot\left(s_{t i}+C_{t i}\right) \\
& \frac{\overline{d_{i}}}{Z \cdot \overline{f_{t i}}}+\overline{p_{i}} \cdot\left(\overline{s_{t i}}+\overline{C_{t i}}\right) \leq \overline{t_{t i}}
\end{aligned}
$$

$$
\leq \frac{\overline{d_{i}}}{Z \cdot \overline{e_{t i}}}+\overline{p_{i}} \cdot\left(\overline{s_{t i}}+\overline{C_{t i}}\right)
$$

$$
i=1,2, \cdots, n-1
$$

$$
\begin{aligned}
& \frac{d_{i}}{Z \cdot h_{t i}} \\
& \quad \leq \frac{d_{i}}{d_{i+1}}\left[t_{t i+1}-p_{i+1} \cdot\left(s_{t i+1}+C_{t i+1}\right)\right] \\
& \quad-\left[t_{t i}-p_{i} \cdot\left(s_{t i}+C_{t i}\right)\right] \leq \frac{d_{i}}{Z \cdot g_{t i}} \\
& \frac{\overline{d_{i}}}{Z \cdot \overline{h_{t i}}} \quad \overline{\overline{d_{i}}}\left[\overline{t_{t i+1}}-\overline{p_{i+1}} \cdot\left(\overline{s_{t i+1}}+\overline{C_{t i+1}}\right)\right] \\
& \quad-\left[\overline{t_{t i}}-\overline{p_{i}} \cdot\left(\overline{s_{t i}}+\overline{C_{t i}}\right)\right] \leq \frac{\overline{d_{i}}}{Z \cdot \overline{g_{t i}}} \\
& \quad i=1,2, \cdots, n-2
\end{aligned}
$$

$f_{t i}\left(\overline{f_{t i}}\right)$ and $e_{i}\left(\overline{e_{i}}\right)$ are the upper and lower boundaries of tram speed between intersections $i$ and $i+1$ in outbound (inbound) direction, respectively. $p_{i}\left(\overline{p_{i}}\right)$ equals 1 when there exists a station between intersections $i$ and $i+1$, and the value would be 0 if there is no station between intersections $i$ and $i+1 . s_{t i}\left(\overline{s_{t i}}\right)$ is the dwell time at the station located between intersections $i$ and $i+1$ in outbound (inbound) direction, which is determined by equation $(20) . C_{t i}\left(\overline{C_{t i}}\right)$ is the time loss caused by tram deceleration and acceleration at the station between intersections $i$ and $i+1$ in outbound (inbound) direction, and it is calculated by equation (21). $h_{t i}\left(\overline{h_{t i}}\right)$ and 
$g_{t i}\left(\overline{g_{t i}}\right)$ are the cap and floor of tram speed variation between the adjacent sections before and after intersections $i+1$ in outbound (inbound) direction, respectively.

$$
\begin{aligned}
& s_{t i}=\left[t_{s}+\max \left(Q_{b i}^{\prime} \cdot \lambda_{b}, Q_{a i}^{\prime} \cdot \lambda_{a}\right)\right] \cdot \frac{1}{Z} \\
& Q_{b i}^{\prime}=\frac{Q_{b i}}{n_{t b}} \cdot \xi_{b i} \\
& Q_{a i}^{\prime}=\frac{Q_{a i}}{n_{t a}} \cdot \xi_{a i} \\
& \overline{s_{t i}}=\left[t_{s}+\max \left(\overline{Q_{b i}^{\prime}} \cdot \lambda_{b}, \overline{Q_{a i}^{\prime}} \cdot \lambda_{a}\right)\right] \cdot \frac{1}{Z} \\
& \overline{Q_{b i}^{\prime}}=\frac{\overline{Q_{b i}}}{n_{t b}} \cdot \overline{\xi_{b i}} \\
& \overline{Q_{a i}^{\prime}}=\frac{\overline{Q_{a i}}}{n_{t a}} \cdot \overline{\xi_{a i}} \\
& \quad i=1,2, \cdots, n-1
\end{aligned}
$$

$t_{s}$ is the door operation time. $Q_{b i}^{\prime}\left(\overline{Q_{b i}^{\prime}}\right)$ and $Q_{a i}^{\prime}\left(\overline{Q_{a i}^{\prime}}\right)$, respectively, are the numbers of boarding and alighting passengers at the busiest door when tram stops at the station between intersections $i$ and $i+1$ in outbound (inbound) direction. $\lambda_{b}$ and $\lambda_{a}$ are the average boarding and alighting time for each passenger, respectively. $Q_{b i}\left(\overline{Q_{b i}}\right)$ and $Q_{a i}\left(\overline{Q_{a i}}\right)$ denote the maximum numbers of boarding and alighting passengers at the station according to the historical demand, respectively. $n_{i b}$ and $n_{i a}$ are the numbers of tram doors for boarding and alighting, respectively. $\xi_{b i}\left(\overline{\xi_{b i}}\right)$ and $\xi_{a i}\left(\overline{\xi_{a i}}\right)$ are the nonuniform coefficients of passenger demands at different doors for boarding and alighting, defined as the ratios of the maximum demand to average demand.

$$
\begin{aligned}
& C_{t 1}=\frac{a_{1} \cdot Z \cdot\left(t_{t 1}-s_{t 1}\right)-\sqrt{a_{1}^{2} \cdot Z^{2} \cdot\left(t_{t 1}-s_{t 1}\right)^{2}-2 a_{1} \cdot d_{1}}}{2 \cdot a_{1} \cdot Z} \\
& C_{t i}=\left(\frac{v_{t i}}{2 \cdot a_{1}}-\frac{v_{t i}}{2 \cdot a_{2}}\right) \cdot \frac{1}{Z} \\
& C_{t n-1} \\
& =\frac{a_{2} \cdot Z \cdot\left(t_{t n-1}-s_{t n-1}\right)-\sqrt{a_{2}^{2} \cdot Z^{2} \cdot\left(t_{t n-1}-s_{t n-1}\right)^{2}+2 a_{2} \cdot d_{n-1}}}{2 \cdot a_{2} \cdot Z} \\
& \overline{C_{t 1}}=\frac{a_{2} \cdot Z \cdot\left(\overline{t_{t 1}}-\overline{s_{t 1}}\right)-\sqrt{a_{2}^{2} \cdot Z^{2} \cdot\left(\overline{t_{t 1}}-\overline{s_{t 1}}\right)^{2}+2 a_{2} \cdot \overline{d_{1}}}}{2 \cdot a_{2} \cdot Z} \\
& \overline{C_{t i}}=\left(\frac{\overline{v_{t i}}}{2 \cdot a_{1}}-\frac{\overline{v_{t i}}}{2 \cdot a_{2}}\right) \cdot \frac{1}{Z} \\
& \overline{C_{t n-1}} \\
& =\frac{a_{1} \cdot Z \cdot\left(\overline{t_{t n-1}}-\overline{s_{t n-1}}\right)-\sqrt{a_{1}^{2} \cdot Z^{2} \cdot\left(\overline{t_{t n-1}}-\overline{s_{t n-1}}\right)^{2}-2 a_{1} \cdot \overline{d_{n-1}}}}{2 \cdot a_{1} \cdot Z}
\end{aligned}
$$

$a_{1}$ and $a_{2}$ are the acceleration and deceleration at stations, in the unit of $\mathrm{m} / \mathrm{s}^{2} . v_{t i}$ is tram speed between intersections $i$ and $i+1$, which is determined by equation (22).

$$
\begin{aligned}
& v_{t i}=\frac{a_{1} \cdot a_{2} \cdot Z \cdot\left(t_{t i}-s_{t i}\right) \pm \sqrt{a_{1}^{2} \cdot a_{2}^{2} \cdot Z^{2} \cdot\left(t_{t i}-s_{t i}\right)^{2}-2 a_{1} \cdot a_{2} \cdot d_{i} \cdot\left(a_{1}+a_{2}\right)}}{a_{1}+a_{2}} \\
& \overline{v_{t i}}=\frac{a_{1} \cdot a_{2} \cdot Z \cdot\left(\overline{t_{t i}}-\overline{s_{t i}}\right) \pm \sqrt{a_{1}^{2} \cdot a_{2}^{2} \cdot Z^{2} \cdot\left(\overline{t_{t i}}-\overline{s_{t i}}\right)^{2}-2 a_{1} \cdot a_{2} \cdot \overline{d_{i}} \cdot\left(a_{1}+a_{2}\right)}}{a_{1}+a_{2}}
\end{aligned}
$$

Finally, in order to satisfy the turnaround plan for trams, the difference of tram travel time between inbound and outbound directions has to be limited in a reasonable range. The constraint is denoted by equation (23):

$$
\left|\sum_{i=1}^{n-1}\left(t t_{i}-\overline{t t_{i}}\right)\right| \leq \varphi
$$

where $\varphi$ is a positive integer related to tram timetable.

In summary, the proposed signal coordinate model consists of objective function (1), constraints (2)-(6), and constraints (9)-(23).

\section{Case Studies}

4.1. Parameters and Results. Case studies on a tramline section in China were conducted to verify the effectiveness of the proposed model. Figure 5 exhibits the relative locations of intersections and tram stations. During the peak hours, all the intersections operate with 180 -second cycle time. The peak hour traffic volume and existing signal timings are shown in Table 1.

Table 2 provides the parameters of the proposed model in case studies. LINGO is applied to attain the solutions of the proposed model and the TRAMBAND model. The green waves and their bandwidths at different intersections are given in Figure 6 and Table 3.

As shown in Figure 6(a), the green bandwidth generated by the proposed model increases at the intersections located at $1300 \mathrm{~m}$ and $670 \mathrm{~m}$ for inbound vehicles, due to the releasing of symmetrical and same width constraints on the green wave band. Allowing variations of green wave bandwidth at different road segments can enlarge the utilization of 


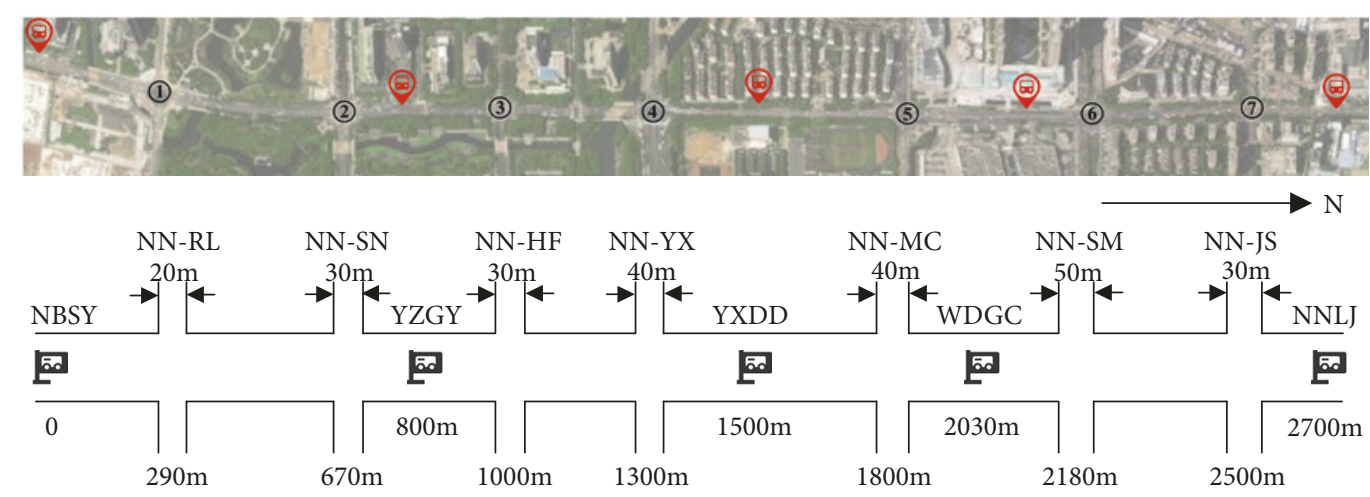

FIGURE 5: Location information of intersections and stations in the tramline.

TABle 1: Traffic volume at intersections and existing signal timing along the tramline.

\begin{tabular}{|c|c|c|c|c|c|c|c|c|c|c|c|c|c|}
\hline \multirow{2}{*}{\multicolumn{2}{|c|}{ Intersection }} & \multicolumn{3}{|c|}{ Traffic flow (pcu/h) } & \multicolumn{2}{|c|}{ Split (s) } & \multirow{2}{*}{\multicolumn{2}{|c|}{ Intersection }} & \multicolumn{3}{|c|}{ Traffic flow (pcu/h) } & \multicolumn{2}{|c|}{ Split (s) } \\
\hline & & Left & Through & Right & Left & Through & & & Left & Through & Right & Left & Through \\
\hline \multirow{4}{*}{$\mathrm{J} 1$} & South & 10 & 720 & 88 & 45 & 98 & \multirow{4}{*}{$\mathrm{J} 5$} & South & 160 & 1008 & 60 & 27 & 90 \\
\hline & North & 276 & 800 & 44 & 45 & 98 & & North & 112 & 624 & 168 & 27 & 90 \\
\hline & East & 104 & 5 & 372 & & 37 & & East & 140 & 470 & 214 & 23 & 40 \\
\hline & West & 20 & 8 & 16 & & 37 & & West & 162 & 370 & 74 & 23 & 40 \\
\hline \multirow{4}{*}{$\mathrm{J} 2$} & South & 68 & 984 & 96 & 45 & 80 & \multirow{4}{*}{ J6 } & South & 174 & 978 & 362 & 25 & 86 \\
\hline & North & 316 & 796 & 2 & 45 & 80 & & North & 206 & 532 & 58 & 25 & 86 \\
\hline & East & 188 & 36 & 212 & & 55 & & East & 340 & 408 & 410 & 34 & 35 \\
\hline & West & 16 & 16 & 16 & & 55 & & West & 244 & 712 & 72 & 34 & 35 \\
\hline \multirow{4}{*}{$\mathrm{J} 3$} & South & 396 & 664 & 24 & 37 & 90 & \multirow{4}{*}{ J7 } & South & 156 & 952 & 258 & 26 & 77 \\
\hline & North & 176 & 504 & 20 & 37 & 90 & & North & 44 & 614 & 82 & 26 & 77 \\
\hline & East & 56 & 540 & 120 & 13 & 40 & & East & 72 & 122 & 120 & 36 & 41 \\
\hline & West & 156 & 432 & 108 & 13 & 40 & & West & 362 & 514 & 132 & 36 & 41 \\
\hline \multirow{4}{*}{$\mathrm{J} 4$} & South & 128 & 1056 & 60 & 21 & 90 & & & & & & & \\
\hline & North & 140 & 596 & 176 & 21 & 90 & & & & & & & \\
\hline & East & 20 & 772 & 132 & 26 & 43 & & & & & & & \\
\hline & West & 264 & 812 & 80 & 26 & 43 & & & & & & & \\
\hline
\end{tabular}

TABLE 2: Value of parameters in the proposed model.

\begin{tabular}{lccccc}
\hline Parameter & Value & Parameter & Value & Parameter & Value \\
\hline$q$ & 4 & $e_{t i}\left(\overline{e_{t i}}\right)$ & $20(\mathrm{~km} / \mathrm{h})$ & $a_{1}$ & $1\left(\mathrm{~m} / \mathrm{s}^{2}\right)$ \\
$e_{i}\left(\bar{e}_{i}\right)$ & $30(\mathrm{~km} / \mathrm{h})$ & $f_{t i}\left(\overline{f_{t i}}\right)$ & $60(\mathrm{~km} / \mathrm{h})$ & $a_{2}$ & $-1.5\left(\mathrm{~m} / \mathrm{s}^{2}\right)$ \\
$f_{i}\left(\bar{f}_{i}\right)$ & $60(\mathrm{~km} / \mathrm{h})$ & $h_{t i}\left(\overline{h_{t i}}\right)$ & $-14.4(\mathrm{~km} / \mathrm{h})$ & $\varphi$ \\
$b_{t}\left(\bar{b}_{t}\right)$ & $1 / 9$ & $g_{t i}\left(\overline{g_{t i}}\right)$ & $14.4(\mathrm{~km} / \mathrm{h})$ & $s_{t i}\left(\bar{s}_{t i}\right)$ & $7 / 20$ \\
\hline
\end{tabular}

TABLE 3: Vehicle green bandwidth by the proposed model and TRAMBAND model.

\begin{tabular}{lcr}
\hline Outbound (Inbound) bandwidth & The proposed model & TRAMBAND model \\
\hline$b_{1}\left(\overline{b_{1}}\right)$ & $20 \mathrm{~s}(38.6 \mathrm{~s})$ & $37 \mathrm{~s}(29.4 \mathrm{~s})$ \\
$b_{2}\left(\overline{b_{2}}\right)$ & $20 \mathrm{~s}(27.7 \mathrm{~s})$ & $37 \mathrm{~s}(29.4 \mathrm{~s})$ \\
$b_{3}\left(\overline{b_{3}}\right)$ & $20 \mathrm{~s}(27.7 \mathrm{~s})$ & $37 \mathrm{~s}(29.4 \mathrm{~s})$ \\
$b_{4}\left(\overline{b_{4}}\right)$ & $28.4 \mathrm{~s}(20 \mathrm{~s})$ & $37 \mathrm{~s}(29.4 \mathrm{~s})$ \\
$b_{5}\left(\overline{b_{5}}\right)$ & $33.5 \mathrm{~s}(20 \mathrm{~s})$ & $37 \mathrm{~s}(29.4 \mathrm{~s})$ \\
$b_{6}\left(\overline{b_{6}}\right)$ & $54.6 \mathrm{~s}(20 \mathrm{~s})$ & $37 \mathrm{~s}(29.4 \mathrm{~s})$ \\
$b_{7}\left(\overline{b_{7}}\right)$ & $73.8 \mathrm{~s}(20 \mathrm{~s})$ & $37 \mathrm{~s}(29.4 \mathrm{~s})$ \\
\hline
\end{tabular}




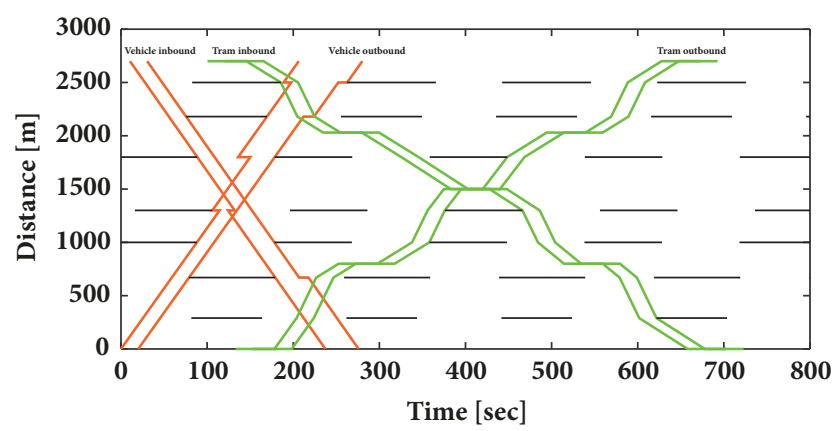

(a) Proposed model in this study

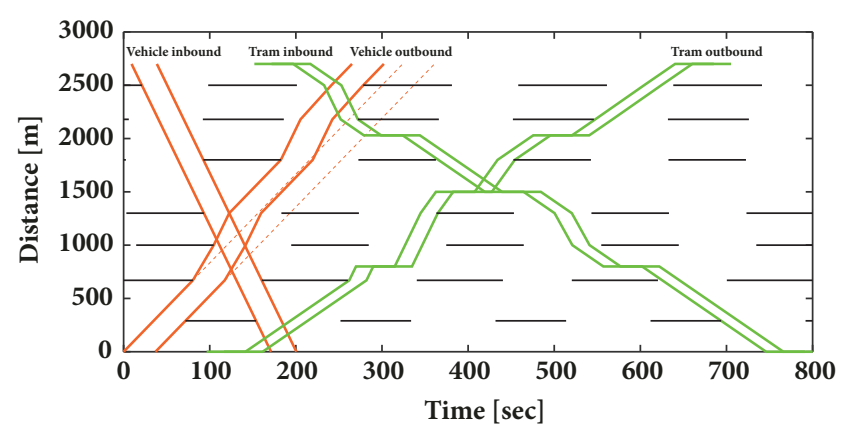

(b) TRAMBAND model

FIgURE 6: Time-space diagram of the proposed model and TRAMBAND model.

green time at some intersections. In contrast, TRAMBAND produced a green band with fixed bandwidth for vehicles, which may reduce the vehicle throughput at intersections. Moreover, the green bandwidth by the proposed model does not decrease, which benefits to eliminate the invalid part of green wave.

With TRAMBAND model, the recommended speed for vehicles to follow the green wave in outbound direction varies at different sections, shown in Figure 6(b). However, most drivers, in practice, are unable to strictly follow the changing recommended speed, which might deteriorate the actual effect of TRAMBAND model. If travelling at a constant speed, the vehicles used to follow the green wave band will meet red signals, as shown in Figure 6(b) where the dash line conflicts with red signals. With the proposed model, the recommended speed to follow the green wave is constant, which may reduce the theoretical bandwidth for vehicles compared to TRAMBAND model as shown in Table 3. Nevertheless, the proposed model is more applicable for most drivers with respect to the current human driving, and it might practically perform even better than the TRAMBAND model which will be discussed in Section 4.2.

The recommended speed for trams to follow green wave in the proposed model is lower than that of TRAMBAND model in the sections with active stations. The reason is that the time loss of braking and motoring caused by tram stopping at stations has been considered in the proposed model, which leads to a lower travel speed.

4.2. Model Performance Evaluation. To compare the performance of the proposed model and TRAMBAND, this study used the microscopic traffic simulation software VISSIM to evaluate the timing plans generated by the two models. In the simulation, tram departs every 180 seconds and tram timetable follows the centre line of green wave band. On evaluation of the timing plan created by the proposed model, vehicles travel at the constant speed recommended by the green wave. For TRAMBAND model, two scenarios were simulated: (1) vehicle speed always matches the speed recommended by the green wave, which is changing from road sections to sections; (2) all vehicles drive at a constant speed (i.e., the speed recommended for the first road segment).
Figure 7 compares the tram and vehicle delays as well as vehicle throughput at the study intersections.

In Figure 7(a), the average tram delay from the TRAMBAND model is $13.15 \mathrm{~s} / \mathrm{pcu}$, regardless of vehicle driving speed. It is much longer than the tram delay $0.01 \mathrm{~s} / \mathrm{pcu}$ from the proposed model, which is caused by the speed limit of tram crossing intersections. The reason is that trams are more likely to hit red signals at some intersections with TRAMBAND model, which does not include the time loss of deceleration and acceleration at tram stations. For example, as shown in Figure 6(b), the green wave band of outbound trams is very close to red signals at the fourth and fifth intersections. The time loss caused by pulling in and out of stations 1 and 2 shifts the real tram trajectory to the right side and trams meet red signals at the fourth and fifth intersections located at 1300 $\mathrm{m}$ and $1800 \mathrm{~m}$.

The average vehicle delay with the proposed model is $26.39 \mathrm{~s} / \mathrm{pcu}$, which is slightly longer than $26.23 \mathrm{~s} / \mathrm{pcu}$ vehicle delay from the TRAMBAND model with dynamic driving speed. This is because the vehicle green bandwidth from the proposed model is a little bit narrower than the green band from the TRAMBAND, due to the constraint that the recommended speed for vehicles should keep constant along the corridor in the proposed model. However, the practical effect of the solution by TRAMBAND model is close to the simulation results with the constant driving speed, because most drivers are reluctant to change vehicle speed at different sections. In this case, the actual average vehicle delay would be $26.99 \mathrm{~s} / \mathrm{pcu}$, which is $2.22 \%$ higher than the solution from the proposed model, shown as Figure 7(b).

The vehicle throughput based on the proposed model is slightly lower than the result from the TRAMBAND model with dynamic driving speed, shown as Figure 7(c). However, the vehicle throughput from the proposed model is $4.45 \%$ larger than the TRAMBAND model result with constant driving speed. The trend of vehicle throughput matches with the vehicle delay results.

4.3. Sensitive Analysis. Tram headway, station dwell time, and traffic volumes are crucial simulation parameters closely related to the model performance. This section aims to analyse the performance stability of the proposed model when these parameters are altered. The performance comparisons 


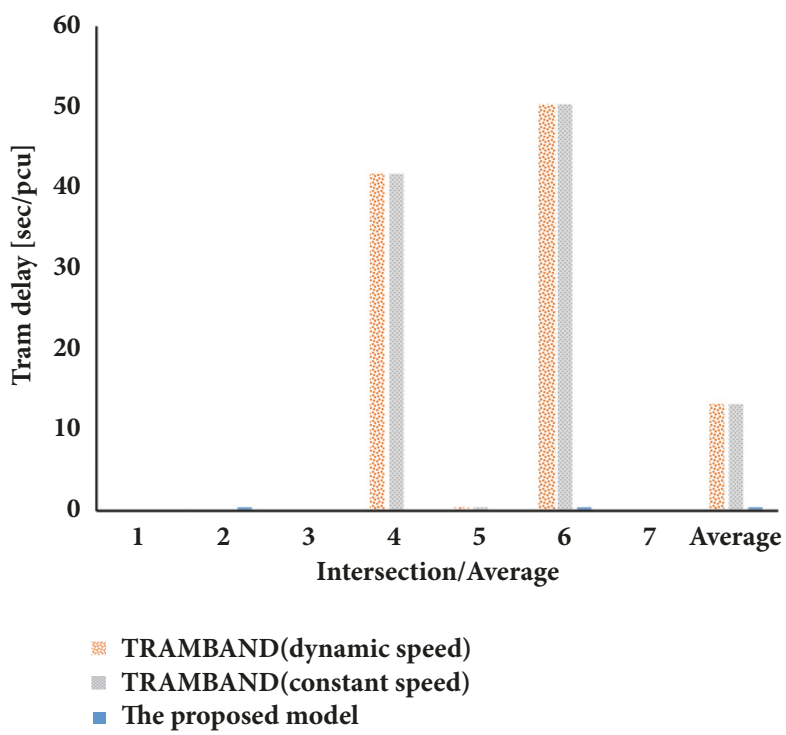

(a) Tram delays

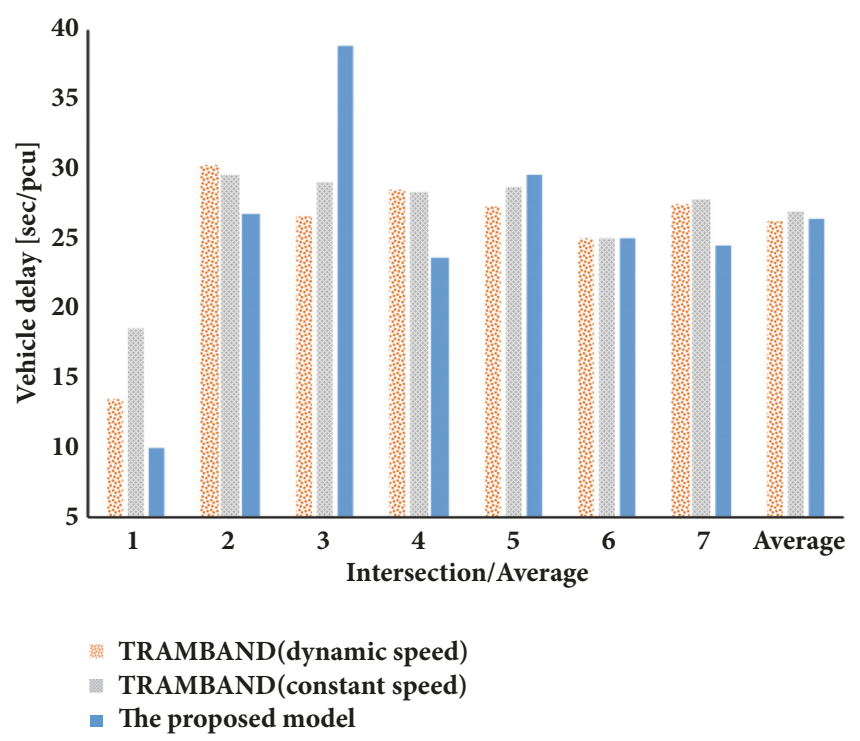

(b) Vehicle delays

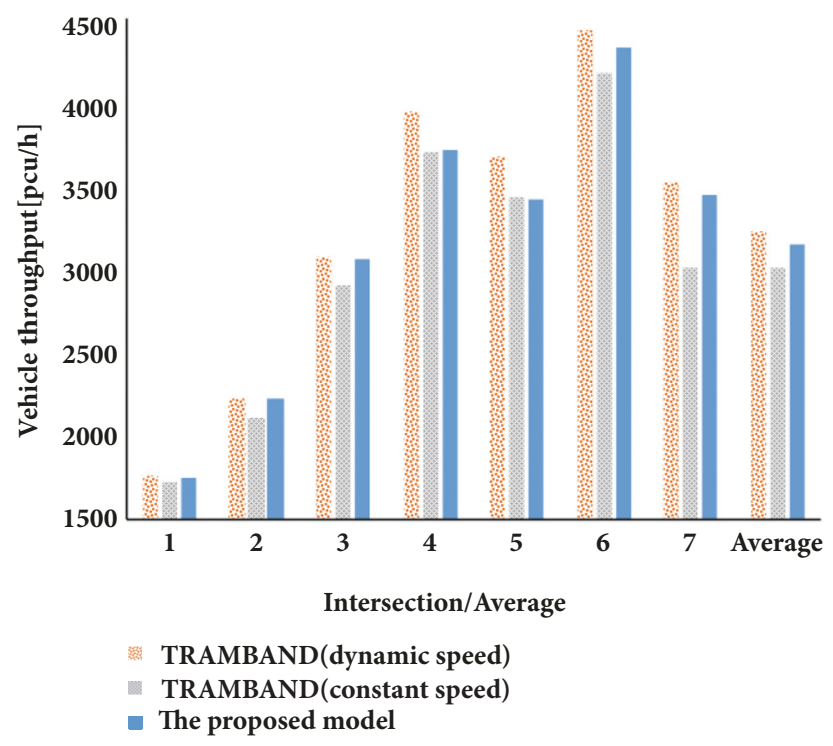

(c) Throughput of vehicles

FIGURE 7: Results comparison between the proposed model and TRAMBAND model.

between the proposed model and the TRAMBAND model under different simulation parameters are shown in Figure 8.

Figure 8 (a) shows that the average tram delay from the proposed model is always smaller than that from the TRAMBAND model with different tram headways (for example, 90 $\mathrm{s}, 180 \mathrm{~s}$, and $360 \mathrm{~s}$ ). The tram delay is close to 0 when the tram departs every $180 \mathrm{~s}$ or $360 \mathrm{~s}$. The average delay is 8.54 $\mathrm{s} / \mathrm{pcu}$ when the tram headway is $90 \mathrm{~s}$ which is shorter than the traffic signal cycle time $180 \mathrm{~s}$. This is because it is unable to create green waves for all trams when the tram headway is not the multiple of signal cycle time. In other words, the tram headway must be multiple of the traffic signal cycle time to realize green wave for all trams at all intersections.

In real practice, tram dwell time at a station depends on the number of boarding and alighting passengers. Because of the fluctuation of passenger demand, the actual dwell time of trams at stations might deviate from the schedule. This study assumed there is only one disturbed station where tram actual dwell time is different from the schedule. Each station except for the last one was in turn chosen as the disturbed station in simulations, because the fluctuation of passenger demand could happen to any one of these stations. For every possible actual dwell time (for example, $30 \mathrm{~s}, 45$ $\mathrm{s}$, and $60 \mathrm{~s}$ ), the average tram delay of all simulations with different disturbed stations is shown in Figure 8(b). The results indicate that the average tram delay from the proposed model is smaller than the result of TRAMBAND model in all the dwell time ranges. Therefore, the proposed model is less sensitive to the dwell time compared to the TRAMBAND model. 


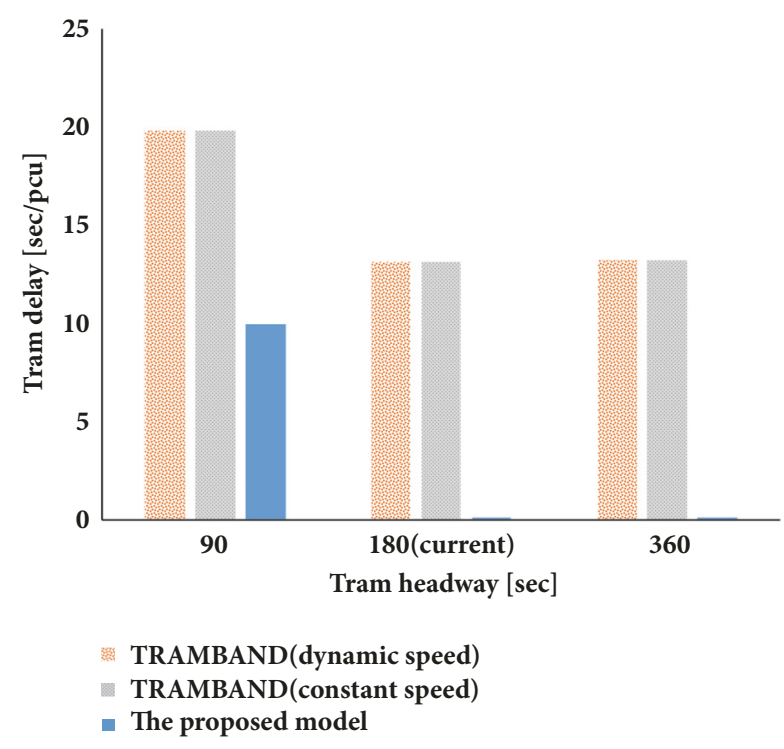

(a) Tram delays with different headways

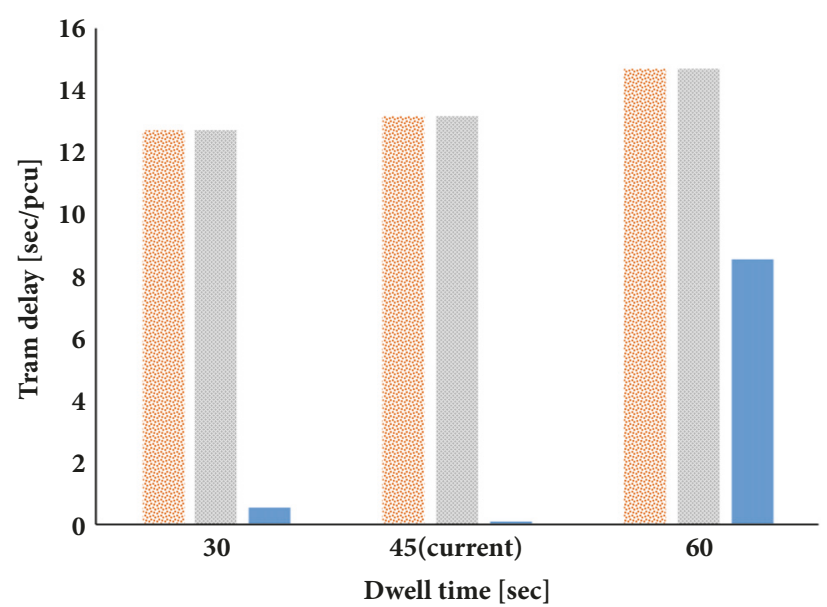

TRAMBAND(dynamic speed)

TRAMBAND(constant speed)

- The proposed model

(b) Tram delays with different dwell time

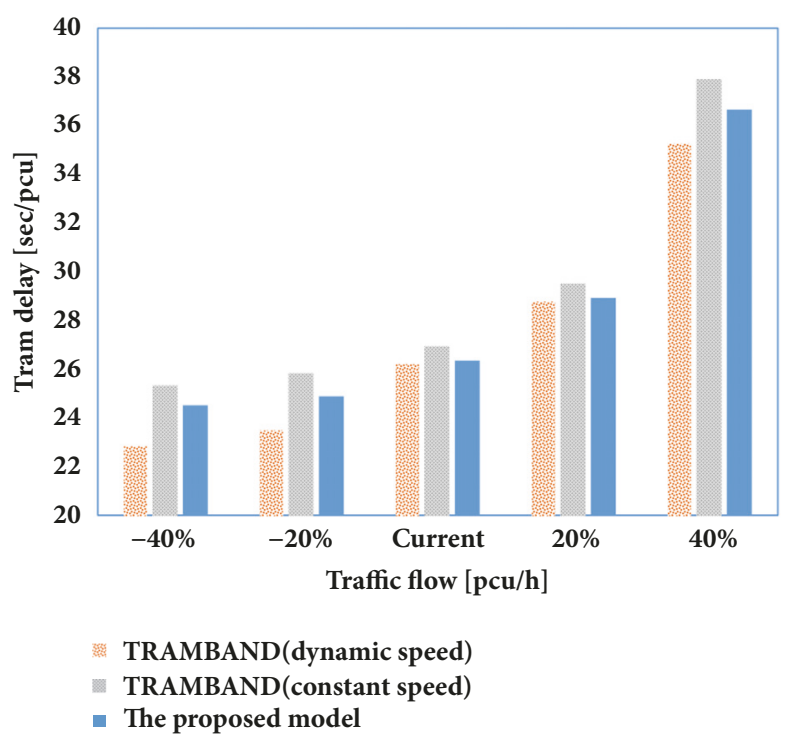

(c) Vehicle delays with different traffic volumes

FIGURE 8: Sensitivity analysis on delays between the proposed model and TRAMBAND model.

The traffic volume also affects the model performances. The average vehicle delay increases with the increasing traffic volumes, as shown in Figure 8(c). The average vehicle delay of the proposed model solution is longer than that from the TRAMBAND model with dynamic driving speed. Nevertheless, it is smaller than that from the TRAMBAND model with constant driving speed, which is in line with the results in Section 4.2. In other words, the practical effect of the solution by TRAMBAND model is not as good as that of the proposed model.

\section{Conclusions}

Prioritizing traffic signals for trams crossing intersections improves the service level of tram operation, but this may result in longer vehicle delays at intersections. Among the available signal priority strategies, the passive priority has been widely adopted to realize the green wave control of trams, with relatively low cost and insignificant impact on vehicle and pedestrian traffic. The performance of passive priority in reducing vehicle delays at intersections is closely related to the intersection signal timing plan along the tramline. To this end, this paper proposed a mathematical model to find the optimal signal time plan at intersections along a tramline, with full consideration of the tram and vehicle driving behaviours. The objective is to maximize the valid bandwidth of green wave for vehicles, while providing the tram with green band to cross the intersections without stops. 
The proposed model has been tested on a tramline in China. In comparison with the traditional TRAMBAND model, the proposed model is able to reduce the average tram delays by $13.14 \mathrm{~s} / \mathrm{pcu}$ and the average vehicle delays by $2.22 \%$. Besides, the solution by the proposed model also enables $4.45 \%$ increment of vehicle throughput at intersections compared to that of the TRAMBAND model. Extensive simulations confirm the performance stability of the proposed model with major sensitive factors including tram dwell time at stations, tram headways, and traffic volumes. The results also suggest that the tram headway must be multiple of the traffic signal cycle time to create more feasible green wave for all trams at all intersections along the tramline.

It is worthy to mention that the proposed model fixed the green wave speed of vehicles so as to adapt to the current human driving of vehicles. In the future, when Intelligent Vehicle Infrastructure Cooperative Systems are available, the vehicles could exactly follow the changing recommended speed. In further studies, we will revise our model by relaxing the constraint on the fixed green wave speed with respect to the Intelligent Vehicle Infrastructure Cooperative Systems. Additionally, tram operation is also a critical factor in realizing green wave control of trams in practice. With inappropriate operation, trams may still meet red traffic lights and stop at the intersection even with a well-designed green wave band. Therefore, the integrated optimization on traffic signal coordination and tram operation will also be explored in future studies.

\section{Data Availability}

All data used to support the findings of this study are available from the corresponding author upon request.

\section{Conflicts of Interest}

The authors declare that they have no conflicts of interest.

\section{Acknowledgments}

This research is funded by the Fundamental Research Funds for the Central Universities (2018JBM025, 2018YJS088), the National Natural Science Foundation of China (71571016), and Public Welfare Technology Application Research Project of Zhejiang Province of China (2016C33256).

\section{References}

[1] M. Mesbah, M. Sarvi, G. Currie, and M. Saffarzadeh, "Policymaking tool for optimization of transit priority lanes in Urban network," Transportation Research Record, vol. 2197, no. 1, pp. 54-62, 2010.

[2] M. Mesbah, M. Sarvi, and G. Currie, "Optimization of transit priority in the transportation network using a genetic algorithm," IEEE Transactions on Intelligent Transportation Systems, vol. 12, no. 3, pp. 908-919, 2011.

[3] A. Skabardonis, "Control strategies for transit priority," Transportation Research Record, vol. 1727, no. 1584, pp. 20-26, 2000.
[4] J. Hu, B. B. Park, and Y.-J. Lee, "Coordinated transit signal priority supporting transit progression under Connected Vehicle Technology," Transportation Research Part C: Emerging Technologies, vol. 55, pp. 393-408, 2015.

[5] P. Mirchandani, A. Knyazyan, L. Head, and W. Wu, "An approach towards the integration of bus priority, traffic adaptive signal control, and bus information/scheduling systems," Computer-Aided Scheduling of Public Transport, vol. 505, pp. 319-334, 2001.

[6] D. Sermpis, P. Papadakos, and K. Fousekis, "Tram priority at signal-controlled junctions," Proceedings of the Institution of Civil Engineers-Transport. Thomas Telford Ltd, vol. 165, no. 2, pp. 87-96, 2012.

[7] X. Hu, B. Long, and X. Zhu, "Signal-planning optimization for bus priority signal intersections on the basis of green loss equilibrium," Journal of Highway and Transportation Research and Development, vol. 10, no. 2, pp. 59-67, 2016.

[8] J. Shi, Y. Sun, P. Schonfeld, and J. Qi, "Joint optimization of tram timetables and signal timing adjustments at intersections," Transportation Research Part C: Emerging Technologies, vol. 83, pp. 104-119, 2017.

[9] W. Ma and X. Yang, "A passive transit signal priority approach for bus rapid transit system," in Proceedings of the IEEE Intelligent Transportation Systems Conference (ITSC '07), pp. 413-418, Seattle, WA, USA, October 2007.

[10] W. J. Ma, K. L. Head, and Y. H. Feng, "Integrated optimization of transit priority operation at isolated intersections: a person-capacity-based approach," Transportation Research Part C: Emerging Technologies, vol. 40, pp. 49-62, 2014.

[11] Y. Jeong and Y. Kim, "Bus coordination model for passive signal priority," in Proceedings of the Eastern Asia Society for Transportation Studies, vol. 8, pp. 233-238, Eastern Asia Society for Transportation Studies, 2011.

[12] J. Zhao, W. Ma, and P. Li, "Optimal design of midblock crosswalk to achieve trade-off between vehicles and pedestrians," Journal of Transportation Engineering, vol. 143, no. 1, Article ID 04016003, 2016.

[13] Z. Yuan, H. Xu, and P. Zhou, "Development of novel vehicle operation control system elementary prototype for modern tram," in Electrical, Information Engineering and Mechatronics, vol. 138, pp. 1781-1788, Springer, London, UK, 2012.

[14] M. Conrad, F. Dion, and S. Yagar, "Real-time traffic signal optimization with transit priority: Recent advances in the signal priority procedure for optimization in real-time model," Transportation Research Record, no. 1634, pp. 100-109, 1998.

[15] F. Dion and B. Hellinga, "A rule-based real-time traffic responsive signal control system with transit priority: application to an isolated intersection," Transportation Research Part B: Methodological, vol. 36, no. 4, pp. 325-343, 2002.

[16] F. Ahmed and Y. E. Hawas, "An integrated real-time traffic signal system for transit signal priority, incident detection and congestion management," Transportation Research Part C: Emerging Technologies, vol. 60, pp. 52-76, 2015.

[17] K. Ling and A. Shalaby, "Automated transit headway control via adaptive signal priority," Journal of Advanced Transportation, vol. 38, no. 1, pp. 45-67, 2003.

[18] W. Ma, W. Ni, L. Head, and J. Zhao, "Effective coordinated optimization model for transit priority control under arterial progression," Transportation Research Record, vol. 2366, no. 1, pp. 71-83, 2013.

[19] S. Shu, J. Zhao, and Y. Han, "Signal timing optimization for transit priority at near-saturated intersections," Journal of 
Advanced Transportation, vol. 2018, Article ID 8502804, 14 pages, 2018.

[20] J. T. Morgan and J. D. C. Little, "Synchronizing traffic signals for maximal bandwidth," Operations Research, vol. 12, no. 6, pp. 896-912, 1964.

[21] J. D. C. Little, “The synchronization of traffic signals by mixedinteger linear programming," Operations Research, vol. 14, no. 4, pp. 568-594, 1966.

[22] J. D. C. Little, M. D. Kelson, and N. H. Gartner, "MAXBAND: a program for setting signal on arteries and triangular network," Transportation Research Record, no. 795, pp. 40-46, 1981.

[23] N. H. Gartner, S. F. Assman, F. Lasaga, and D. L. Hou, "A multi-band approach to arterial traffic signal optimization," Transportation Research Part B: Methodological, vol. 25, no. 1, pp. 55-74, 1991.

[24] C. Stamatiadis and N. H. Gartner, "MULTIBAND-96: a program for variable-bandwidth progression optimization of multiarterial traffic networks," Transportation Research Record, vol. 1554, no. 1, pp. 9-17, 1996.

[25] N. H. Gartner and C. Stamatiadis, "Arterial-based control of traffic flow in urban grid networks," Mathematical and Computer Modelling, vol. 35, no. 5-6, pp. 657-671, 2002.

[26] C. Zhang, Y. Xie, N. H. Gartner, C. Stamatiadis, and T. Arsava, "AM-band: an asymmetrical multi-band model for arterial traffic signal coordination," Transportation Research, Part C: Emerging Technologies, vol. 58, pp. 515-531, 2015.

[27] G. Y. Dai, H. Wang, and W. Wang, "A bandwidth approach to arterial signal optimisation with bus priority," Transportmetrica A: Transport Science, vol. 11, no. 7, pp. 579-602, 2015.

[28] G. Y. Dai, H. Wang, and W. Wang, "Signal optimization and coordination for bus progression based on MAXBAND," KSCE Journal of Civil Engineering, vol. 20, no. 2, pp. 890-898, 2016.

[29] Y. Jeong and Y. Kim, “Tram passive signal priority strategy based on the MAXBAND model," KSCE Journal of Civil Engineering, vol. 18, no. 5, pp. 1518-1527, 2014.

[30] Y. Zhou, S. Jia, B. Mao, T. K. Ho, and W. Wei, "An arterial signal coordination optimization model for trams based on modified AM-BAND," Discrete Dynamics in Nature and Society, vol. 2016, Article ID 5028095, 10 pages, 2016. 


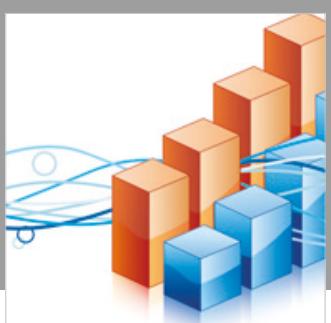

Advances in

Operations Research

\section{-n-m}
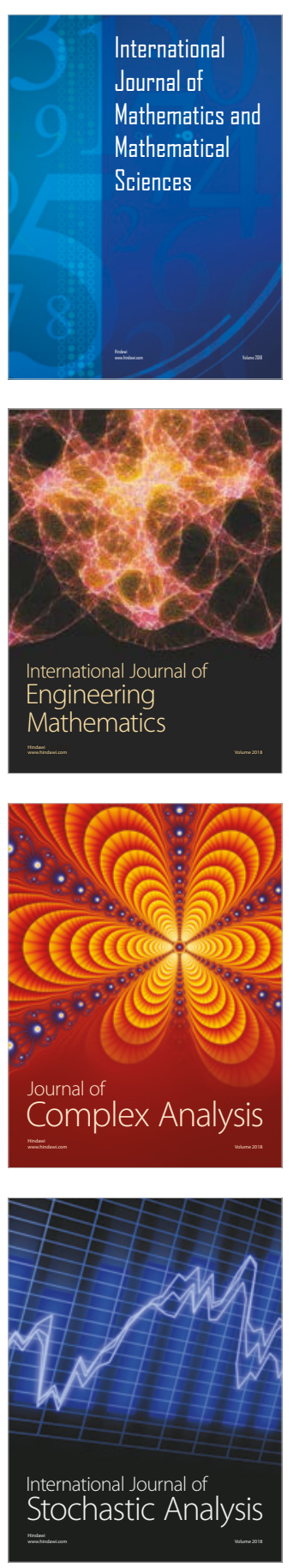
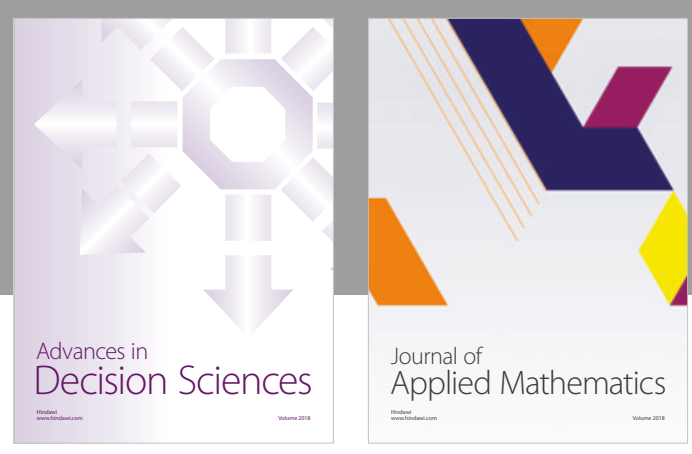

Journal of

Applied Mathematics
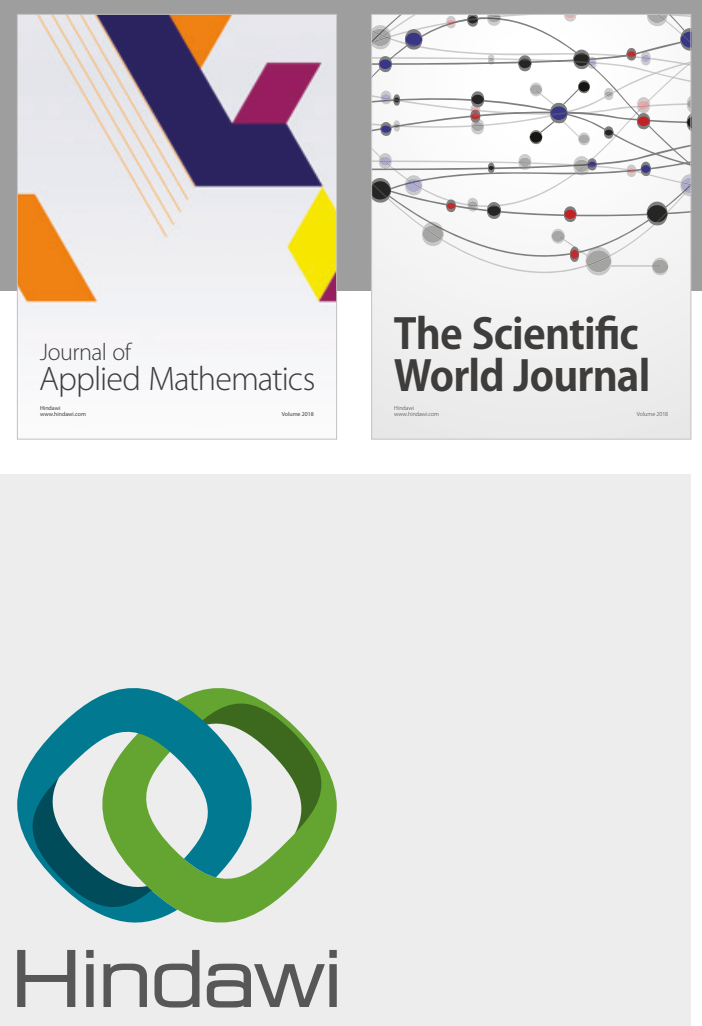

Submit your manuscripts at

www.hindawi.com

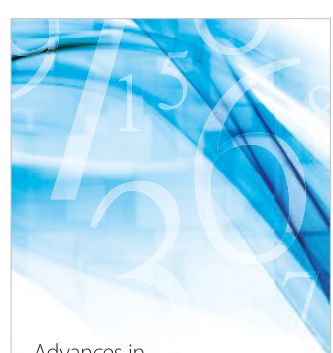

Advances in
Numerical Analysis
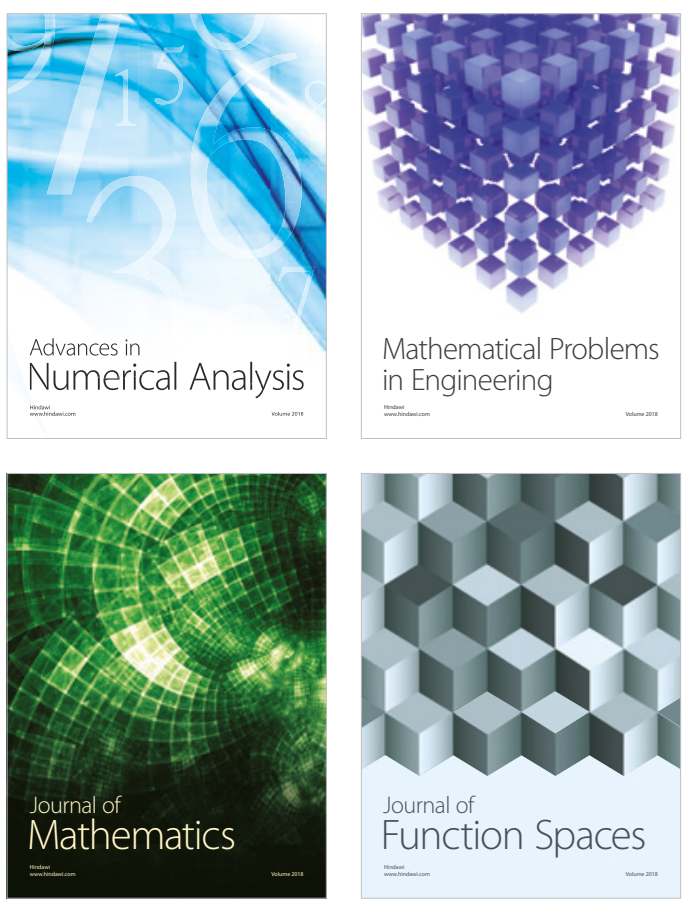

Mathematical Problems in Engineering

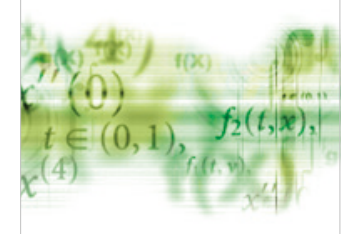

International Journal of

Differential Equations

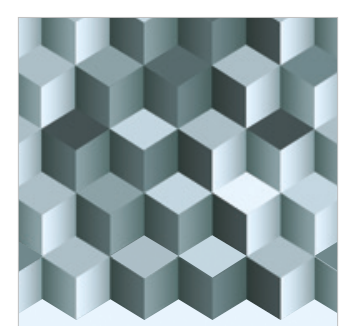

Journal of

Function Spaces
The Scientific

World Journal

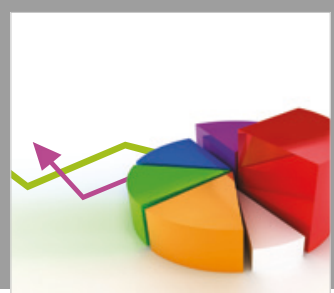

Journal of

Probability and Statistics
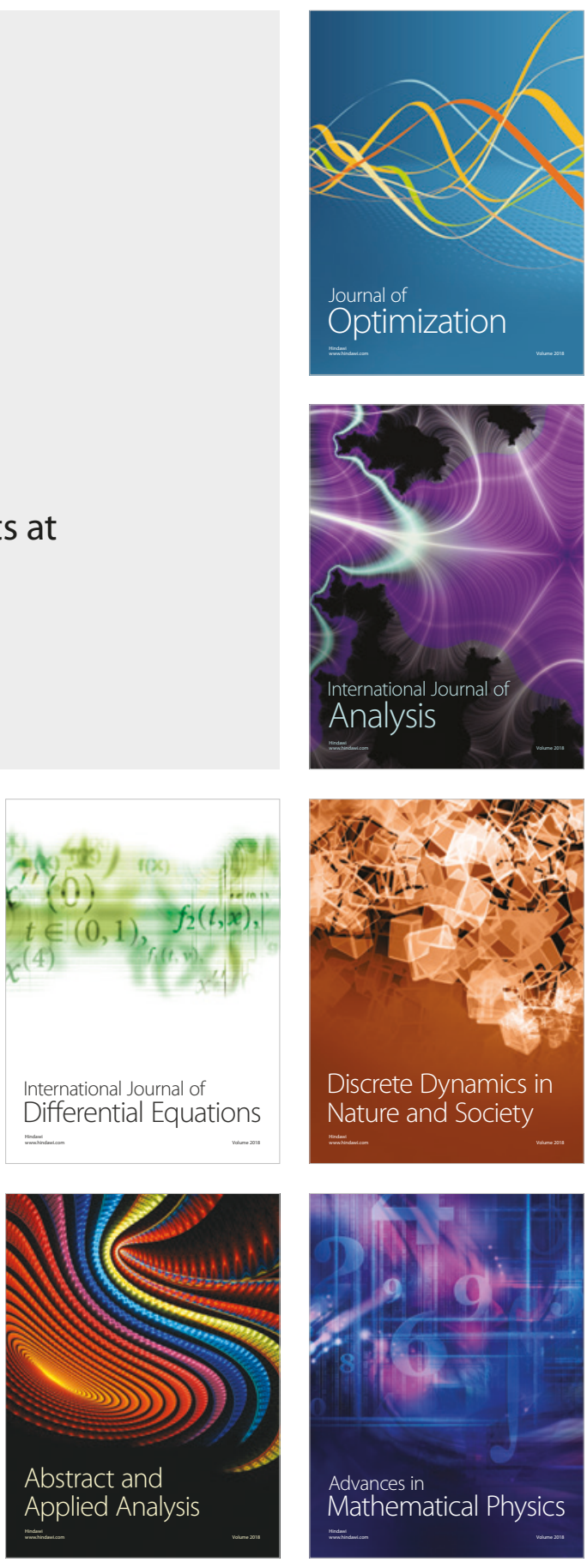